\title{
Contactless Electrical Resistance of 2D Materials Using a Rutile Resonator
}

\author{
David Arcos1, Patrick Krkotić2,3, Joan M. O’Callaghan2, Montse Pont3, Lluís Ametller1, \\ Núria Ferrer-Anglada1 \\ 1 Department of Physics, Universitat Politècnica de Catalunya (UPC), Campus Nord, Barcelona, Spain \\ 2 CommSensLab, TSC Department, Universitat Politècnica de Catalunya (UPC), Campus Nord, Barcelona, Spain \\ 3 ALBA Synchrotron - CELLS, Cerdanyola del Valles, Barcelona, Spain
}

Received ZZZ, revised ZZZ, accepted ZZZ

Published online ZZZ (Dates will be provided by the publisher.)

Keywords Graphene, bucky paper, rutile resonator, high frequency conductivity.

Measuring the electrical surface resistance of 2D materials without contact can provide a method for obtaining their intrinsic characterisation. The aim of this paper is to show that a rutile dielectric resonator (RDR) can be used to measure the electrical surface resistance of conducting coatings deposited on substrates, at the resonance frequency. Moreover, it is known that the substrate exerts a strong influence capable of intrinsically modify the properties of the $2 \mathrm{D}$ materials, as found in graphene. The RDR method is used for different

1. Introduction The characterization of electrical and optical properties of $2 \mathrm{D}$ materials such as graphene at microwave frequency range has attracted a lot of attention in recent years. This is due to the many applications to engineering, such as graphene-based devices, graphene field effect transistors, graphene antennas and graphene microstrip attenuators, among others [1].

The electrical resistance $R_{s}$ (and hence the electrical conductivity) of a conducting coating can be determined using a cavity end-wall replacement method [2]. Using a low-loss, high permittivity dielectric to load the resonator enables the measurement of electrical resistance $R_{s}$ of a conducting coating at a microwave range of frequencies, determined from the changes of the quality factor $\mathrm{Q}$ when the cavity is modified by the sample [ $3-5]$. This nondestructive evaluation technique is based on the interaction of electromagnetic waves with the sample under investigation. Therefore, to obtain the electrical properties samples of metals $(\mathrm{Cu}, \mathrm{Mo}, \mathrm{Ti}$, brass), carbon nanotubes (bucky paper), a film of compacted graphene flakes, a film of compacted graphene oxide flakes and graphene obtained by CVD on different substrates ( $\mathrm{SiO} 2 / \mathrm{Si}$, quartz and PET). The results show that reasonable values can be obtained for thin conducting materials with a thickness of not less than a few micrometers. In the case of graphene grown on a substrate, the presence of graphene is clearly detected but the resistivity value cannot be extracted.

Copyright line will be provided by the publisher

of 2D materials, the thickness of the sample has a clear importance, especially if the film thickness (typically a few micrometers) becomes comparable or smaller than the skin depth of the material. In such a case, electromagnetic fields penetrate through the coating and the sample can no longer be treated as a bulk material, which leads to a multi-layer system. The objective of this work is to explore the dielectric resonator technique for the characterization of different thin graphene-based samples at microwave frequencies.

1.1. Rutile dielectric resonator (RDR) The RDR is composed of a closed metallic body housing a rutile $\left(\mathrm{TiO}_{2}\right)$ cylinder with a height of $3 \mathrm{~mm}$ and a diameter of $4 \mathrm{~mm}$, shielded axially by a pair of identical samples (squares of $12 \times 12 \mathrm{~mm}$ ) to be examined and fixed with a pair of brass blocks, as shown in Figure 1 [3]. The rutile has a high permittivity $(\varepsilon r \approx 100)$ and a very low loss factor $(\tan (\delta) \leq 10$ - 
4), and its small size, compared with the total size of the cavity, ensures that the electromagnetic field in the lateral walls is effectively neglected for the TE011 resonance mode. The electromagnetic field in the cavity is produced by a pair of semi-rigid coaxial cables with a loop at the end for magnetic coupling, as shown in the cross-section in Figure 2 .
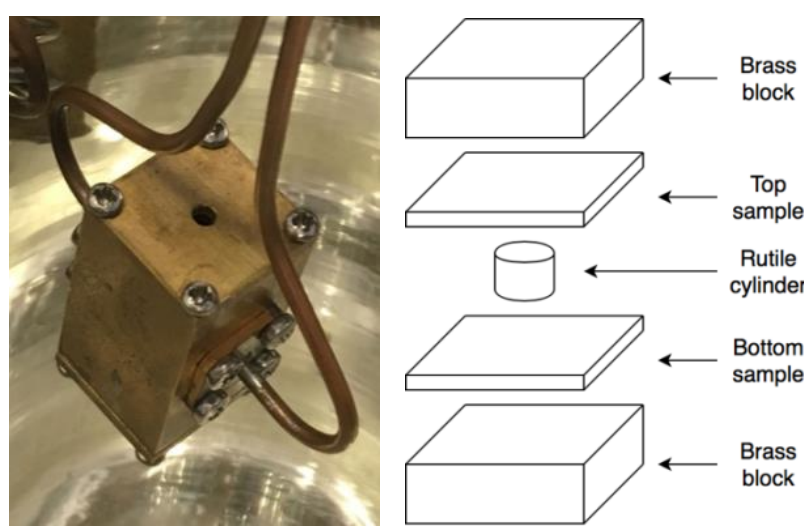

Figure 1. Photograph of the RDR and a scheme of the inner layers.

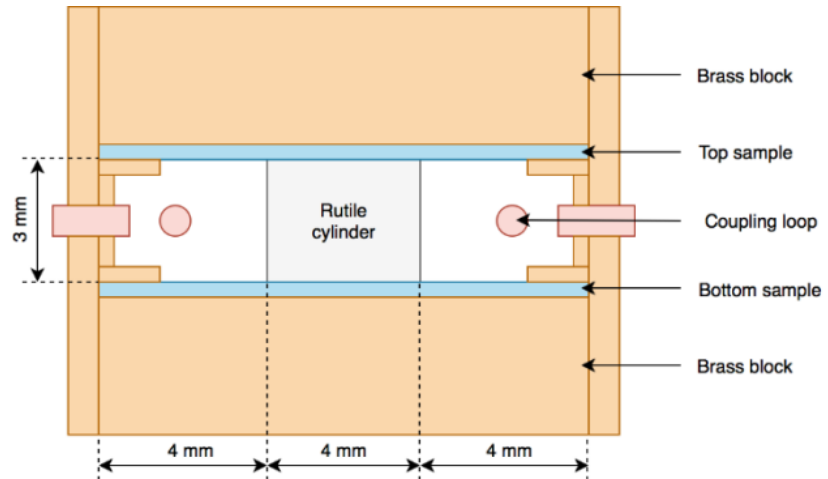

Figure 2. Scheme of the RDR cross-section.

1.2. Measurement methodology A vector network analyzer was used to measure the quality factor, which is defined as the ratio between the resonance frequency of the resonant cavity and the $3 \mathrm{~dB}$-bandwidth. It is also defined as a measure of the ratio between the stored energy and the energy dissipated in the resonator. The surface resistances $R_{S_{i}}$ of the surfaces enclosing the whole cavity are directly related to the quality factor $Q$ in the following way [6]:

$$
\frac{1}{Q}=\sum_{i} \frac{R_{S_{i}}}{R_{G S_{i}}}+p \cdot \tan (\delta)
$$

Where:

- $\tan (\delta)=1.2496 \cdot 10^{-4}$ is the loss tangent of rutile at room temperature modeling dielectric losses.

- $\quad p \sim 1$ is the ratio of the energy stored in the dielectric to the energy stored in the entire resonator.
- $R_{G S}=242.529 \Omega$ is the geometrical factor of the closing plates of the resonator, which has been determined analytically, numerically and experimentally [3]

- The sum takes into account the losses of the individual metal surfaces noted through the index ' $i$ '. Note that the lateral walls can be neglected.

We determine the resistivity, $\rho$, of the material by using the well-known relation between square surface resistance, $R_{\mathrm{s}}$, and resistivity, at the resonance frequency, $f_{0}$, given by:

$$
R_{s}=\frac{\rho}{\delta_{s}}, \quad \delta_{s}=\sqrt{\frac{\rho}{\pi \cdot f_{0} \cdot \mu_{0}}} \quad \Rightarrow \quad \rho=\frac{R_{s}{ }^{2}}{\pi \cdot f_{0} \cdot \mu_{0}}
$$

Here, $\delta$ s is the skin depth, which can be seen as a measure of how closely electrical current flows along the surface of a material, and $\mu_{0}=4 \pi \cdot 10^{-7} \mathrm{H} \mathrm{m}_{-1}$ the permeability in vacuum.

When only one target sample is available (instead of having two identical samples enclosing the rutile cylinder), equation 1 can be rewritten as:

$$
\frac{1}{Q_{c o m b}}=\frac{R_{S 1}+R_{S 2}}{R_{G S}}+p \cdot \tan (\delta)
$$

where we consider the surface resistances of the two different materials, e.g. measuring the surface resistance of a full metal resonator, for example, brass or copper in the first stage, this known surface resistance can then be used to determine the unknown surface resistance of graphene using equation 3.

Equation 2 assumes that the thickness of the sample is at least three times greater than the skin depth of the material If that condition is not true, as in the case of the samples with monolayers, the electromagnetic field passes through the sample and the effective surface resistance of the sample plus the substrate is measured instead. In this case, the problem becomes that of a multi-layer system, and the properties of the substrate (the second layer) also play a role and need to be determined in order to estimate the actual surface resistance of the graphene sample, depending on its thickness.

\subsection{Sample characterization of $2 \mathrm{D}$ materials $A$} set of carbon-based samples, graphene and carbon nanotubes (CNT) were analyzed in order to compare the results. $S_{1}$ is a bucky paper sample made of chemical vapor deposition (CVD) single wall carbon nanotubes. All other samples are graphene. $S_{2}$ and $S_{3}$ are compacted graphene flakes obtained by reducing graphene oxide flakes. $\mathrm{S}_{4}$ is a film of graphene oxide flakes, unreduced. Samples $\mathrm{S}_{5}, \mathrm{~S}_{6}$ and $\mathrm{S}_{7}$ are obtained (at Graphenea) by CVD on different substrates: $\mathrm{S}_{5}$ is graphene on quartz, $\mathrm{S}_{6}$ is graphene on PET and $\mathrm{S}_{7}$ is graphene on $\mathrm{SiO}_{2} / \mathrm{Si}$. 
In order to fit inside the resonant cavity, the geometry of all the samples analyzed in this work consists of a square with sides of approximately $11.5 \mathrm{~mm}$ and of a certain thickness, so the thickness of each sample is the most remarkable geometrical difference. These samples are summarized in Table 1.

The thickness of the samples was measured in different ways. For samples $S_{1}$ and $S_{2}$, a caliper with a resolution of $5 \mu \mathrm{m}$ was used. For samples $\mathrm{S}_{3}$ and $\mathrm{S}_{4}$, we used a microscope focusing on the ground plate as a reference. By changing the height of the lens until the focus is on the surface of the sample, we can estimate the thickness of the sample. The error arises due to the adjustment limitations of the lens.

Table 1. Summary of samples analyzed in the current study. Sample identifications are used for later reference.

\begin{tabular}{cccc}
\hline Material & Substrate & Sample & Thickness $[\mu \mathrm{m}]$ \\
\hline Bucky paper (CNT) & - & $S_{1}$ & $30 \pm 5$ \\
Graphene flakes & - & $S_{2}$ & $20 \pm 5$ \\
Graphene flakes & - & $S_{3}$ & $10 \pm 1$ \\
Graphene oxide & - & $S_{4}$ & $13 \pm 1$ \\
Graphene & Quartz & $S_{5}$ & $<10 \mathrm{~nm}$ \\
Graphene & PET & $S_{6}$ & $<10 \mathrm{~nm}$ \\
Graphene & $\mathrm{Si} / \mathrm{SiO}_{2}$ & $S_{7}$ & $<10 \mathrm{~nm}$ \\
\hline
\end{tabular}

In order to check the quality of graphene samples, Raman spectroscopy (excitation laser of $532 \mathrm{~nm}$ and power $0.50 \mathrm{~mW}$ ) was used; see Figures 3 and 4.

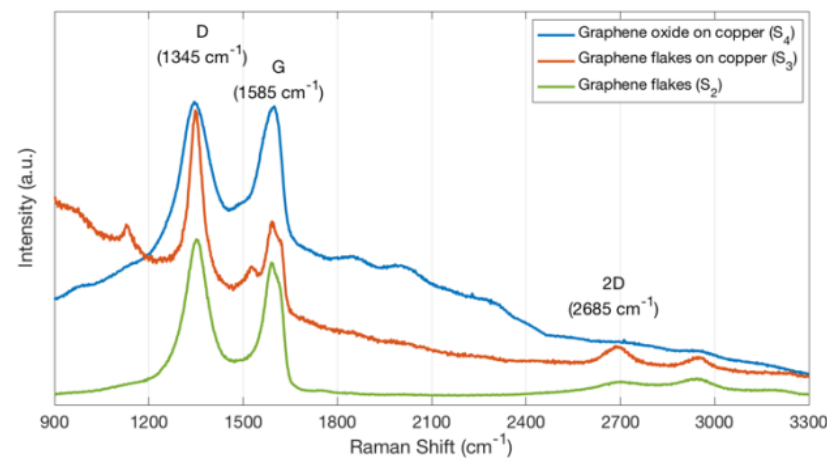

Figure 3. Raman spectra of graphene flake film $\left(\mathrm{S}_{2}\right)$, graphene flake film on copper $\left(\mathrm{S}_{3}\right)$ and graphene-oxide flake film on copper $\left(\mathrm{S}_{4}\right)$.

It is well known that the Raman spectra for graphene is characterized by two main peaks, the D band (at $1345 \mathrm{~cm}-1$ ) the $\mathrm{G}$ band at about $2685 \mathrm{~cm}-1$ ). The D-band is due to defects, as disordered sp3 carbon and dangling bonds, whereas the G-band is due to ordered graphitic carbons as sp2 hybridization [7 - 11]. The relative intensity ratio of these two bands $I_{D} / I_{G}$ is an indication of the graphitization degree of the sample: a low $I_{D} / I_{G}$ ratio corresponding to an elevated graphitization degree, and hence a high electrical conductivity could be expected. This ratio is also correlated inversely to the average size of graphene (sp2) domains [12]. The deconvolution and baseline correction of the Raman spectra for our samples $\mathrm{S}_{2}, \mathrm{~S}_{3}$ (graphene) or $\mathrm{S}_{4}$ (graphene oxide) shows a ratio $I_{D} / I_{G}$ greater than $1\left(I_{D} / I_{G}=1.16\right.$ for $\mathrm{S}_{2}$, 2.02 for $\mathrm{S}_{3}, 1.00$ for $\mathrm{S}_{4}$; see Figure 3). Therefore, we can conclude that a large number of defects exist, either in graphene oxide or in the reduced graphene samples.

A further graphene characteristic peak exists, which is the $2 \mathrm{D}$ band at $2685 \mathrm{~cm}-1$ and characteristic of $\mathrm{sp} 2$ carbons. The relative intensity ratio $I_{2 D} / I_{G}$ is related to the number of graphene layers in few-layer graphene, for a monolayer $I_{2 D} / I_{G} \gg>1$. In our case, only samples S5 (CVD graphene on Quartz) and S7 (CVD graphene on $\mathrm{Si} / \mathrm{SiO} 2$ ) could be monolayer in some regions, while the graphene flake samples are multi-layer. The main difference between the Raman spectra of graphene samples, $S_{2}$ and $S_{3}$, and the graphene oxide sample, $S_{4}$, is the visibility of the $2 \mathrm{D}$ peak in the reduced ones, $S_{2}$ and $S_{3}$. This may be due to a greater disorder on the graphene oxide sample.

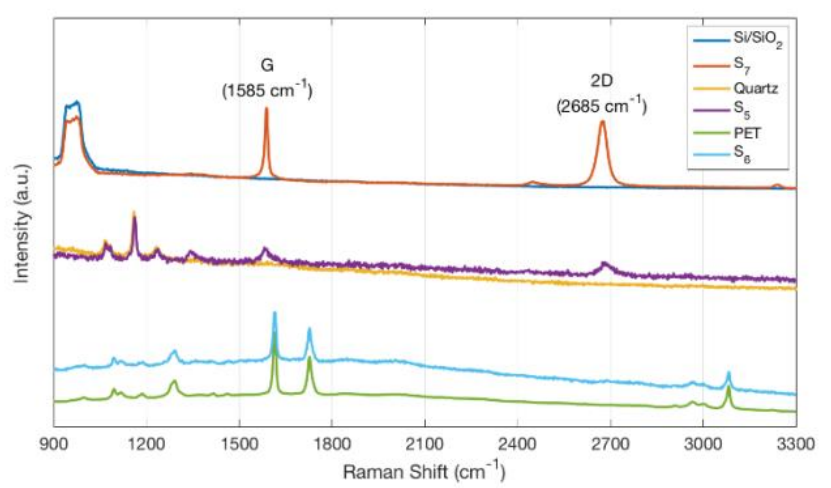

Figure 4. Raman spectra of CVD graphene samples $\mathrm{S}_{5}, \mathrm{~S}_{6}$ and $\mathrm{S}_{7}$ compared with their bare substrates.

Figure 4 shows the Raman spectra of CVD graphene samples on different substrates compared with their bare substrates. The Raman spectra of $\mathrm{S}_{7}$, graphene on $\mathrm{Si}_{/} \mathrm{SiO}_{2}$, is characteristic of a pure graphene sample without defects (the D band is not observed) [7, 10-12]. In the case of $\mathrm{S}_{5}$, graphene on Quartz, the Raman spectra is also characteristic of graphene, but with low intensity. Finally, in the case of S6, graphene on PET, the interference of the substrate hides the graphene characteristic bands, as we observe that the spectra of S6 and bare PET are practically the same.

\section{Experimental results}

2.1. Measurements of common metals We start the analysis with a pair of equal samples of some common metals in order to test the method. The coupling is adjusted for a very low coupling $\left(S_{21}<-40 \mathrm{~dB}\right.$; see Figure 5) to 
assure small errors, and the loaded quality factor can be assumed to be the same as the unloaded. The results obtained are shown in Table 2.

Table 2. Summary of the resonance frequency $f_{0}$, the quality factor $Q$, the surface resistance $R s$, the resistivity obtained $\rho_{m}$ and resistivity found in the literature $\rho l$ [13] for common metal samples.

\begin{tabular}{cccccc}
\hline Material & $\begin{array}{c}f_{0} \\
{[\mathrm{GHz}]}\end{array}$ & $Q$ & $\begin{array}{c}R s \\
{[\mathrm{~m} \Omega]}\end{array}$ & $\begin{array}{c}\rho m \\
{[\mu \Omega \mathrm{cm}]}\end{array}$ & $\begin{array}{c}\rho l \\
{[\mu \Omega \mathrm{cm}]}\end{array}$ \\
\hline Copper & 9.0152 & 2864 & 27.19 & 2.08 & 1.72 \\
Molybdenum & 9.0090 & 2100 & 42.59 & 5.10 & 5.35 \\
Titanium & 9.0105 & 905.0 & 118.8 & 39.7 & 42.0 \\
Brass & 9.0262 & 1742 & 54.46 & 8.32 & $\sim 6-9$ \\
\hline
\end{tabular}

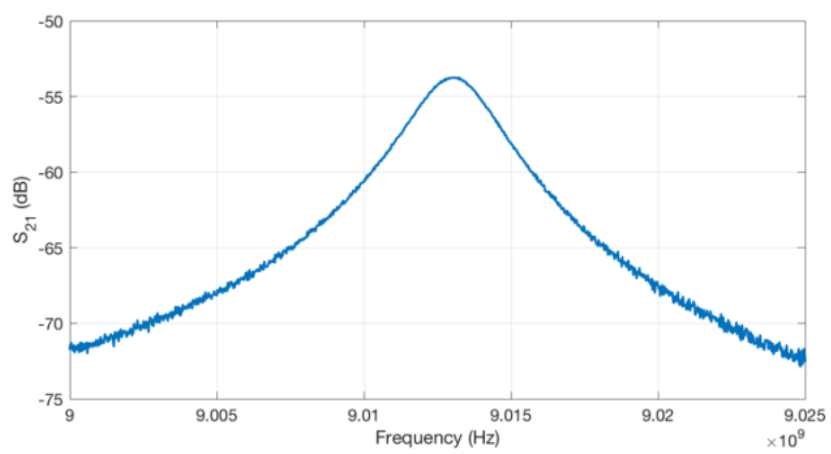

Figure 5. $S_{21}$ parameter measured in the RDR when closed with copper on each side. The resonance frequency measured is $f_{0}=$ $9.0131 \mathrm{GHz}$ and the quality factor is $Q=3052$.

2.2. Measurements of 2D materials We continue the analysis with samples of the 2D materials mentioned in Table 1, and we consider different strategies according to the following conditions:

i) The number of equal samples available (one or two).

ii) The thickness of the samples, $t_{s}$, compared to the skin depth, $\delta$, of the material under study.

iii) The type of substrate (metal or dielectric).

In condition i), we distinguish between two different configurations for the resonator: the metal-sample (just one sample available) and the sample-sample (two identical samples). When we have two identical samples, the measurement process is easier because only a single measurement using the resonator is needed. Should only one sample be available (this is the case for samples $S_{1}$ and $S_{2}$ ), it is necessary to take a preliminary measurement of the factor Q of a full metal resonator (i.e. configuration metalmetal) in order to obtain the Rs of the known metal by using equation 1 . We then replace one of the metal samples with the target sample in order to obtain a new $\mathrm{Q}$ factor value and use it in equation 3 to obtain $R s$ of the target sample.
In condition ii), it is necessary to distinguish between a bulk material $\left(t_{s}>3 \delta_{s}\right)$ and a layered material $\left(t_{s} \leq 3 \delta_{s}\right)$. In the first case, the surface resistance measured is directly related to the resistivity in accordance with equation 2 . However, in the case of thin materials (compared to skin depth), the effective surface resistance is measured instead, and it is necessary to calculate the resistivity of the sample layer using a multi-layer model, in which the effective surface resistance is due to the different layers: the sample layer plus the reference metal and the substrate.

Condition iii) is important in the case of thin layer materials $\left(t_{s} \leq 3 \delta_{s}\right)$ because then, when the substrate is a bulk metal, the electromagnetic field is shielded by the substrate and the effective surface resistance is the combination of the sample and the substrate (2-layer model). In the case of a dielectric substrate (e.g. samples $\mathrm{S}_{5}, \mathrm{~S}_{6}$ and $\mathrm{S} 7$ ), the electromagnetic field reaches the brass block resonator surfaces and the number of layers to be considered in the model is three (sample, dielectric substrate and metal).

For each sample (see Table 1), it is necessary to analyze these conditions, then we are able to divide our measurements into 3 different configurations.

2.2.1. Configuration metal-sample with $t s \gg \delta s$ In this case, since only one sample is available, we need to use a calibration measurement (configuration metal-metal) with a known material (brass or copper) to be able to compare the change in the quality factor. This is the case for the samples $S_{1}$ and $S_{2}$. The results obtained are shown in Table 3. Here, in rows two and four, when using CNT bucky paper or graphene flakes, the relationship between the quality factor and $R s$ is given by equation 3 . To be more precise, the factor $Q$ is due to the combination of bucky paper with brass or graphene flakes with copper.

Table 3. Summary of the resonance frequency $f_{0}$, the quality factor $\mathrm{Q}$, the surface resistance $R_{S}$ and the resistivity $\rho$ obtained for bucky paper and graphene flake samples. For calibration, the values for brass and copper from Goodfellow are shown. Each surface resistance/resistivity value refers to the top sample.

\begin{tabular}{cccccc}
\hline $\begin{array}{c}\text { Top } \\
\text { sample }\end{array}$ & $\begin{array}{c}\text { Bottom } \\
\text { Sample }\end{array}$ & $f_{0}[\mathrm{GHz}]$ & $Q$ & $R_{s}[\mathrm{~m} \Omega]$ & $\rho[\mu \Omega \mathrm{cm}]$ \\
\hline $\begin{array}{c}\text { Brass } \\
\text { S: CNT }\end{array}$ & Brass & 9.0262 & 1742 & 54.5 & 8.32 \\
$\begin{array}{c}\text { Bucky paper } \\
\text { Copper }\end{array}$ & Brass & 8.9285 & 745.2 & 241 & 164 \\
$\begin{array}{c}\text { S2: Graphene } \\
\text { flakes }\end{array}$ & Copper & 9.0131 & 3052 & 24.6 & 1.70 \\
\hline
\end{tabular}

2.2.2. Configuration sample-sample with $t_{s} \sim \delta_{s}$ When the thickness of the sample is comparable with the skin depth of the material, we have to consider a two-layer system. We obtain the resistivity of the sample from the 
effective surface resistance measured, since we know the properties of the metal reference (resistivity and skin depth) and the thickness of the target sample. This has been done for samples $S_{3}$ and $S_{4}$ and the results are summarized in Table 4.

Table 4. Summary of resonance frequencies $f_{0}$ and quality factors $Q$ obtained for bulk copper, graphene flake and graphene oxide flake samples.

\begin{tabular}{ccccc}
\hline Samples & $f_{0}[\mathrm{GHz}]$ & $Q$ & $R_{s}[\mathrm{~m} \Omega]$ & $\rho[\mu \Omega \mathrm{cm}]$ \\
\hline $\begin{array}{c}\text { Copper } \\
\text { Graphene flakes } \\
\text { on Copper }\end{array}$ & 9.0135 & 3001 & 25.2 & 1.79 \\
$\begin{array}{c}\text { Graphene oxide } \\
\text { on Copper }\end{array}$ & 9.9870 & 476.4 & 452 & 576.5 \\
\hline
\end{tabular}

For graphene flakes ( $\mathrm{S} 3$ sample) we obtain a resistivity value which is four times greater than the value obtained for the sample $S_{2}$ in Table 3 . This may be accounted for by the sample quality and roughness. Furthermore, the sample $\mathrm{S}_{2}$ looks brighter than $\mathrm{S}_{3}$, which suggests a better conductivity as it is found experimentally.

Comparison of the $Q$-Value results of the multi-layer system graphene oxide on copper with single layer copper shows that the graphene oxide has such a low conductivity that it is basically invisible at the thickness of few microns. This is not a surprising result, since graphene oxide is an insulator $(\rho>103 \Omega \mathrm{cm})[12,14]$.

\subsubsection{Configuration sample-sample with $t_{s}<<\delta$ s} on dielectric substrate In this case, which is the most common in CVD graphene samples deposited on a substrate (samples $\mathrm{S}_{5}, \mathrm{~S}_{6}$ and $\mathrm{S}_{7}$ ), we wish to point out that an observable difference in quality factor values exists when considering only the bare substrate or when the sample with graphene is on one or both sides of the RDR. The results obtained are shown in Table 5.

It is evident from Table 5 that the monolayer is visible, since the measured quality factor falls notably with the replacement of bare substrates for the graphene coated substrate, both for quartz and PET substrates. It should be taken into account that the thickness of the graphene $(<10$ $\mathrm{nm}$ ) leads to a perturbation of the TE011 mode, since the electromagnetic field is influenced by the thickness of the substrates. It is for this reason the resonance frequency is lower than when the cavity is closed with bulk metals. In the case of the $\mathrm{Si} / \mathrm{SiO}_{2}$ substrate, no resonance is obtained because it is a lossy material and the electromagnetic field decays rapidly.

Table 5. Summary of resonance frequencies $f_{0}$ and quality factors $Q$ obtained for graphene (and bare substrate) samples.

\begin{tabular}{llll} 
Top sample & Bottom sample & $f_{0}[\mathrm{GHz}]$ & $Q$ \\
\hline
\end{tabular}

\begin{tabular}{cccc}
\hline Quartz & Quartz & 8.1336 & 4513 \\
Graphene on Quartz & Quartz & 8.1153 & 267 \\
Graphene on Quartz & Graphene on Quartz & 8.1243 & 154 \\
PET & PET & 8.3200 & 4004 \\
Graphene on PET & PET & 8.4595 & 2605 \\
Graphene on PET & Graphene on PET & 8.4834 & 417 \\
$\mathrm{Si} / \mathrm{SiO}_{2}$ & $\mathrm{Si}_{\mathrm{SiO}}$ & - & - \\
\hline
\end{tabular}

3. Error Estimation For the analysis of the experimental errors involved in equation 1, we consider those arising from the determination of the geometrical factor $(\Delta R G S)$, the uncertainty of the loss tangent $(\Delta \delta)$, and those resulting from the measurement of the quality factor $(\Delta Q)$. Equation 4 shows the effect of all these errors on the uncertainty of the surface resistance $(\Delta R s)$.

$$
R_{S} \pm \Delta R_{S}=\frac{1}{2}\left(R_{G S} \pm \Delta R_{G S}\right)\left(\frac{1}{Q \pm \Delta Q}-p(\tan (\delta) \pm \Delta \delta)\right)
$$

It has been proven in [3] that the spread in measured quality factor for repeated measurements of the same sample is approximately $5 \%$. As may be observed in Figure 6 (titanium sample), the data obtained in this study is quite noisy due to a low coupling. Hence, a conservative approach of a $10 \%$ error in the quality factor has been taken into account in our analysis for a noisy response, as well as for the $\mathrm{Q}$ factor obtained for samples $\mathrm{S}_{1}$ and $\mathrm{S}_{2}$.

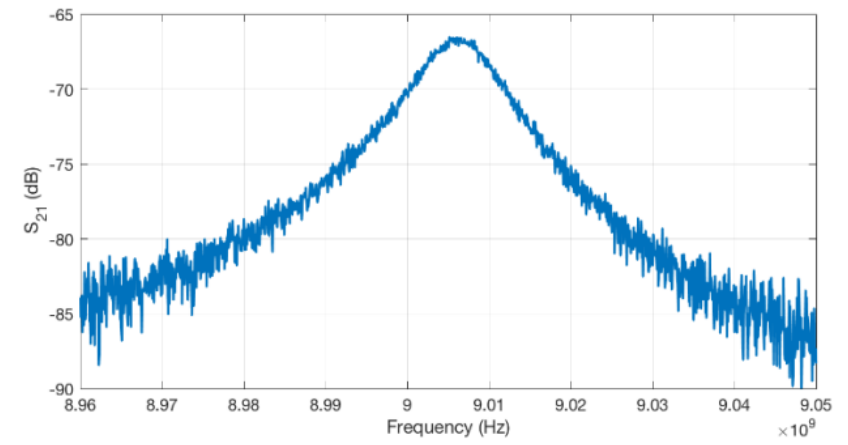

Figure 6. $S_{21}$ parameter measured in the RDR when closed with titanium on each side. The resonance frequency measured is $f_{0}=$ $9.0068 \mathrm{GHz}$ and the quality factor is $Q=953.0$.

For the geometrical factor, RGs, the small discrepancy between the analytical, experimental and numerical determination of its value shows that the error here is negligible. Furthermore, the loss tangent is in the range of 10-4. The relative error in $R S$ is therefore approximately $7 \%$ for metals (values shown in Table 2) and $15 \%$ for samples $\mathrm{S}_{1}$ and $\mathrm{S}_{2}$. The relative error for resistivity is double due to the relation between $R s$ and $\rho$ (see equation 2).

The other source of uncertainty is the thickness of the graphene layer, which is required to determine the surface resistance in the case of the 2-layer system (sample $\mathrm{S}_{3}$ ). 
Measuring the thickness of such a sample is a challenging task. The estimated error of $\pm 1 \mu \mathrm{m}$ in the thickness of the sample can actually lead to a total relative error of $60 \%$ in the estimated conductivity. This significant error is mostly due to the shape of the sample, which is not perfectly flat or uniform.

4. Discussion and conclusions When using two identical samples of common metals, the obtained resistivity values are in good agreement with the reported values for these metals: $\mathrm{Cu}, \mathrm{Mo}, \mathrm{Ti}$ and brass. The resonance appears clearly near to $9 \mathrm{GHz}$ with a $Q$ factor greater than 1000 .

When only one sample is available, with a thickness greater than the skin-depth of the material, it is possible to extract the surface resistance and resistivity of the sample with two measurements: one with two identical metal plates, the other with the same metal and the sample. We obtained reasonable values for the resistivity of carbon nanotubes bucky paper $\left(\mathrm{S}_{1}\right)$ and graphene flake samples $\left(\mathrm{S}_{2}\right)$, similar to other reported values by other authors. In particular, we obtained a very similar value $[12,14]$ for reduced graphene.

With samples of a thickness comparable with the skindepth of the material, it is necessary to apply a multi-layer model. To prove that the thickness of the sample is lower than the skin depth, measurements of the sample were made on two different substrates, which we previously characterized by their electrical properties using our resonator setup. Additionally, it is necessary to determine the thickness of the sample with great precision.

When using CVD monolayer or few-layer graphene, as grown on a substrate, the electromagnetic field is not shielded by the samples, so in our mode a strong perturbation is found, leading to changes in the resonance frequency and quality factor. One may observe the strong influence of the graphene layer on the values obtained for the $Q$ factor, so it is possible to conclude that the method is sensitive to the presence of graphene, and a multi-layer method should be used.

Our experimental setup is in some respects similar to that reported in [4] using a high Q sapphire puck microwave resonator.

The present setup was built for superconducting materials and is unable to increase the coupling by moving the coupling loops inward. When it is used to measure nonsuperconducting materials, the resulting transmission coefficients $\left(S_{21}\right)$ are much lower and the noise increases, mostly when $S_{21}$ falls below $-65 \mathrm{~dB}$ (see Figures 5 and 6). A new setup with adjustable loops is under construction, in order to increase the transmission coefficients and allow higher sensitivity requirements.

Acknowledgements The authors wish to thank the Scie ntific and Technological centers, Universitat de Barcelona (CCiT-UB) services for Raman spectroscopy facilities. We also wish thank Danubia NanoTech Ltd for samples of graphene oxide, $\mathrm{S}_{4}$, and reduced graphene flakes, $\mathrm{S}_{2}, \mathrm{~S}_{3}$, and
V. Skákalová for her interesting comments. This work was supported by the Spanish "Comision Interministerial de Ciencia y Tecnologia” under project MDM2016-0600.

\section{References}

[1] M. Bozzi, L. Pierantoni, S. Bellucci, Radioengineering 2015, 24, 3, 661 .

[2] R. H. Ono, J. C. Booth, F. Stork, C. Wilker, in Advances in Superconductivity $X$ (Eds: I. Hirabayashi, K. Osamura), Springer, Japan 1998, 1407

[3] P. Krkotić, A. Aguasca, J. M. O'Callaghan, 2018 48th European Microwave Conference (EuMC) 2018, 882.

[4] L. Hao, J. Gallop, S. Goniszewski, A. Gregory, O. Shaforost, N. Klein, R. Yakimova, Appl. Phys. Lett. 2013, 103, 123103.

[5] J. Krupka, W. Strupinski, N. Kwietniewski, J. Nanosci. Nanotechnol. 2011, 11, 3358.

[6] D. Kajfez, P. Guillon, Dielectric Resonators, Noble Publishing Corporation, Tucker, GA, 1998.

[7] A. C. Ferrari, J. C. Meyer, V. Scardaci, C. Casiraghi, M. Lazzeri, F. Mauri, S. Piscanec, D. Jiang, K. S. Novoselov, S. Roth, K. A. Geim, Phys. Rev. Lett. 2006, 97, 187401/1.

[8] W. Xu, N. Mao, J. Zhang, Small 2013, 9, 8, 1206.

[9] I. Childres, L. A. Jauregui, W. Park, H. Cao, Y. P. Chen, in New Developments in Photon and Materials Research (Ed: J. I. Jang), Nova Science Publishers, Hauppauge, NY 2013, Ch. 19.

[10] S. Kasap, I. I. Kaya, S. Repp, E. Erdem, Nanoscale Adv. 2019, $1,2586$.

[11] R. Genc, M. O. Alas, E. Harputlu, S. Repp, N. Kremer, M. Castellano, S. G. Colak, K. Ocakoglu, E. Erdem, Sci. Rep. 2017, 7, 11222.

[12] Z. Li, Y. Yao, Z. Lin, K.-S. Moon, W. Lin, C. Wong, J. Mater. Chem. 2010, 20, 4781.

[13] M. Ohring, Engineering Materials Science, Academic Press, San Diego, CA 1995.

[14] X. Xie, Y. Zhou, K. Huang, Front. Chem. (Lausanne, Switz.) 2019, 7,355 . 